\title{
An Efficient Multi-sensing and GSM Equipped Fire Monitoring System
}

\author{
Md. Fahad Bin Zamal ${ }^{\text {* }}$, Shehrin Sayed ${ }^{2}$, Touhid Bhuiyan ${ }^{1}$, Mostafijur Rahman $^{3}$ \\ ${ }^{1}$ Department of Software Engineering, Daffodil International University (DIU), Bangladesh \\ ${ }^{2}$ Department of Electrical \& Electronic Engineering, Bangladesh University of Engineering and Technology (BUET), Bangladesh \\ ${ }^{3}$ School of Computer and Communication Engineering, Universiti Malaysia Perlis (UNIMAP), Malaysia
}

\begin{abstract}
The principal goal of fire monitoring system is to react promptly to a fire and not to misleading particulate signatures produced by nuisance sources. In this paper we proposed a system that not only able to detect and prevent fire at early stages but also capable of interact with surround environment. Recent researches lead us to detect fire by light and heat sensor, image processing, smoke detection mechanism but failed to integrate those in one The advancement on fire detection technologies has been significant over the last few decade due to rapid progress in communication technologies, advances in sensing devices and greater understanding of fire physics. But lack of intelligence among fire monitoring system often failed to make an impact on fire incidents. Our proposed fire monitoring system is incorporated in such a way that can communicate with environment by itself through the help of GSM Modem. Here we introduce an intelligent and advance fire monitoring system that can communicate by itself with fire station and can detect fire at its early stage and extinguish it in the shortest time subject to a few effective factors.
\end{abstract}

\section{Introduction}

Human lives, infrastructure and ecological system is always been threaten by fire and still facing challenges to reduce forged alarms and unable to provide support in evacuation. Rapid progresses can make the communication technologies, advances in sensing devices and establish greater understanding on fire but still we are lagging behind to accumulate those knowledge and technology together. Expensive, complicated and sensitive devices are being used but lack of intelligence is present there.

Most of the fire detection systems use single sensor and operate without any controlling mechanism. Those devices either use temperature sensing or smoke detection concept to identify the presence of fire [1]. But if we use multi sensor detectors, it increases the efficiency in early fire detection as well as it can monitor multiple aspects of a wide variety of fires. Distributed temperature monitoring system is currently used on fire protection system in cities [2]. Another mechanism being used in recent years which is distributed fiber optic temperature measurement system but it has some drawbacks of excessive cable and very high cost [3]. Wireless sensor network in other hand will be a good solution as it is small, easy to maintain, cheap and suitable for large-scale application [4].

This paper studies the design standards and principles of fire detection mechanism, communication mechanism and multiple sensing mechanisms. Multiple sensing mechanisms enhance the accuracy to detect fire incidents and inclusion of stepper motor will provide mitigation of fire in early stage. Microcontroller (ATmega32/Arduino) associated with temperature sensor (LM35) and smoke detector (LDR) will operate to accurately identify the presence of fire. Introduction of GSM modem in this system will make the device capable of communicate with fire stations and localities. The system consists Temperature Detection Module (TDM) where LM-35 sensor being used, Smoke Detection Module (SDM) create by Light Dependent Resister (LDR), Automatic Action Module (AAM) consists stepper motor, and Notification Module (NM) operate with GSM modem.

\section{System Design and Architecture}

\subsection{System Overview}

Our proposed system contains four (4) modules. Those are:
A. Temperature Detection Module (TDM)
B. Smoke Detection Module (SDM)
C. Automatic Action Module (AAM) and
D. Notification Module (NM)

In temperature detection module (TDM) it will always check some parameters before generating a fire alarm. Missing any condition will not trigger the fire alarm which will make the system more accurate and

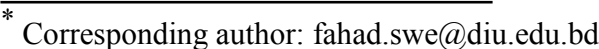


precise. The temperature detection modules first detect temperature that is above the critical temperature and secondly it will check the rate of temperature rises along with the time or not. Here critical temperature (Tc) will be set manually in the microcontroller and it will always aware of the instantaneous value of the temperature (T). If $\mathrm{T}>\mathrm{Tc}$, then the first condition will be satisfied and it will then concentrate on checking whether temperature is rising or not. As an example if temperature for a certain time is $\mathrm{T} 1$. After $\Delta \mathrm{t}$ time the temperature raises to $\mathrm{T} 2$. Then, rate of temperature rise, $r=T 2-T 1 / \Delta t$.If $r$ is very high then the second condition is also satisfied.

Smoke detection module (SDM) will finally check the presence of smoke by the help of smoke detector sensor. If there is smoke, the smoke detector circuit gives a digital ' 1 ' to the microcontroller. Otherwise it gives digital ' 0 '. If these three conditions are fulfilled, then microcontroller turns on the alarm. The Automatic Action Module (AAM) automatically turns on the stepper-motor which is attached to a mechanical system providing water/gas to minimize the effect. In Notification Module (NM) incorporate with GSM modem will send a caution message to nearby residences and also firefighting department with an exact location of the incident.

\subsection{System Architecture}

Figure 1 shows the overall circuit diagram of our proposed fire monitoring system consists Temperature Detection Module (TDM), Smoke Detection Module (SDM), Automatic Action Module (AAM) and Notification Module (NM).

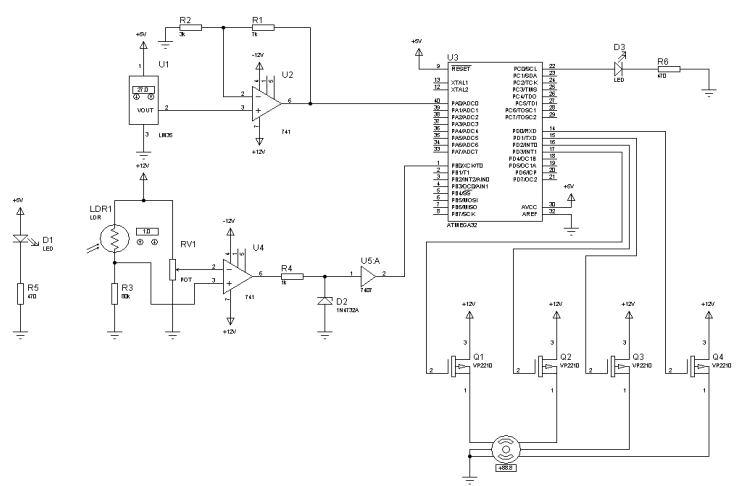

Fig. 1. System Circuit Diagram

\subsection{1: Temperature Detection Module (TDM)}

In our proposed Temperature Detection Module (TDM) we use LM35 sensor for temperature detection mechanism. In case of LM35 which is integrated circuit temperature sensor has an advantage over other linear temperature sensors as there is no requirement of subtract a large voltage from the output. It does not require any external calibration to provide accuracy. An another added advantage is its lower cost. Because of low output impedance, precise calibration, single power supplies, linear output and low cost makes LM35 sensor a better choice over other temperature detection sensors.
It produces output voltages proportional to ${ }^{\circ} \mathrm{C}$ $\left(10 \mathrm{mV} /{ }^{\circ} \mathrm{C}\right)$, so the nominal output voltage is $250 \mathrm{mV}$ at $25^{\circ} \mathrm{C}$ and $1.000 \mathrm{~V}$ at $100^{\circ} \mathrm{C}$. The LM35 is more accurate $\left( \pm 1^{\circ} \mathrm{C}\right.$ from $-55^{\circ} \mathrm{C}$ to $+150^{\circ} \mathrm{C}$ vs. $\pm 3^{\circ} \mathrm{C}$ from $-20^{\circ} \mathrm{C}$ to $+100^{\circ} \mathrm{C}$ ) temperature measuring sensor. Figure 2 shows the overall architecture of Temperature Detection Module (TDM).

In Figure 2 section 1(Red labeled) senses the temperature and converts it to an electrical signal in proportion to the temperature. If the temperature is $\mathrm{T}$ degree Celsius, then the output of the LM35 is given in equation 1 .

$$
\text { Vout }=T \times 10 \mathrm{mV}
$$

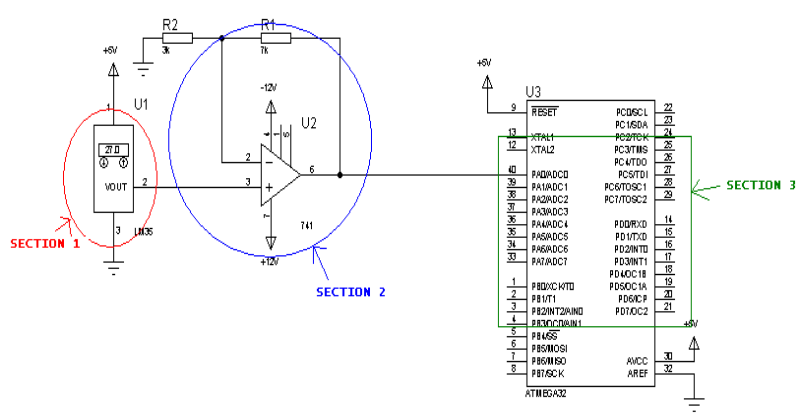

Fig. 2. Temperature Detection Module (TDM)

For example, if the temperature is $30^{\circ} \mathrm{C}$ then output of LM35 is $300 \mathrm{mV}$ or $0.3 \mathrm{~V}$. The working range of $\mathrm{LM} 35$ is $0^{\circ} \mathrm{C}$ to $150^{\circ} \mathrm{C}$. So the range of its output is from $0 \mathrm{~V}$ to $1500 \mathrm{mV}$ or $1.5 \mathrm{~V}$. Figure 3 shows the temperature measuring mechanism by LM35.

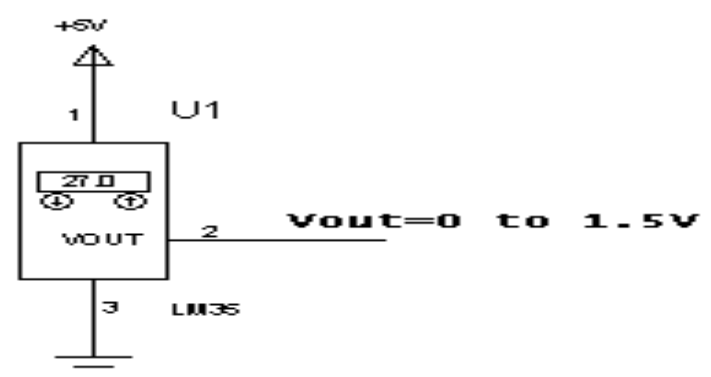

Fig. 3. Temperature Measurement by LM35

The input range of $\mathrm{ADC}$ is $0 \sim 5 \mathrm{~V}$ and the output range of LM35 is $0 \sim 1.5 \mathrm{~V}$. So, the output of LM35 must be amplified to make it suitable for the ADC input. It means we have to convert the 0 to $1.5 \mathrm{~V}$ range into 0 to 5 $\mathrm{V}$ range. In figure 2 section 2 (Blue labeled) we use a non-inverting amplifier which is capable to do this operation. By calculating we found that we have to use a non-inverting amplifier of gain $=5 / 1.5=3.3$. In our proposed system, we adjust the two resistors R1 and R2 in such a way that it will give a gain of 3.3. For getting desire gain we have to select the resistance of $\mathrm{R} 2$ in $33 \mathrm{~K} \Omega$ and $\mathrm{R} 1$ in $10 \mathrm{~K} \Omega$. So setting the value of $\mathrm{R} 1$ and $\mathrm{R} 2$ in that value $(10 \mathrm{~K} \Omega, 33 \mathrm{~K} \Omega)$ we able to get the desired gain and the non-inverting amplifier doing the rest and gives us the desired range for ADC. 
In Figure 4 section 3 (Green labeled) ADC of microcontroller coverts an analog signal to a digital signal. It takes an analog signal within the range of $0 \mathrm{~V}$ to $5 \mathrm{~V}$. The converted value is stored in a 10bit register in the microcontroller. If the analog input is $0 \mathrm{~V}$ then the value stored in the register is 0 and if the analog input is $5 \mathrm{~V}$ the value stored in the register will be 1023 . If the analog input is between $0 \mathrm{~V}$ and $5 \mathrm{~V}$ then the stored value may be any number between 0 and 1023. For example, if the analog input is $3.2 \mathrm{~V}$, then the stored value in the register is

$$
\text { Register }=1023 / 5 \times 3.2=655
$$

The microcontroller is programmed such that it reads the data from the register and calculates the room temperature. Microcontroller continuously reads the value stored in the register and calculates the temperature which is sensed by the LM35.Suppose, at some point the value stored in the register is 512 . Then the analog input at $\mathrm{ADC}$ ( $\mathrm{ADC} 0$ pin of microcontroller) is:

$$
\mathrm{ADC}=5 / 1023 \times 512=2.502 \mathrm{~V}
$$

This is the output of the non-inverting amplifier. Since the gain of the amplifier is 3.3, the input of the amplifier should be

$$
\text { Output } / \text { Gain }=2.502 / 3.3=0.75 \mathrm{~V} \text {. }
$$

Again, the input of the amplifier is the output of LM35. So, output of the LM35 is $0.75 \mathrm{~V}$ or $750 \mathrm{mV}$.

$$
\text { Temperature }=750 \mathrm{mV} / 10 \mathrm{mV}=75^{\circ} \mathrm{C} .
$$

\subsection{2: Smoke Detection Module (SDM)}

Figure 4 shows the schematic view of our proposed Smoke Detection Module (SDM). In this module LDR (Light Dependent Resistors) is used to examine the presence of smoke. The LDR is selected because of its exciting features, such as: when the light level is low the resistance is high (this prevents current flow into the circuit), when light level is high resistance falls and current flows into the circuit.

In this module a comparator Op-Amp is integrated, which is used to compare two voltages and switches, and its output to indicate which voltage is larger. Here it compares the voltage value we adjust in the Potentiometer and the voltage we get when the LDR is starting to conduct. So when comparator compare both voltage value and found that voltage value in the LDR section is larger than it switches $\mathrm{a}+\mathrm{V}_{\mathrm{s}}$ in the circuit. This $+\mathrm{V}_{\mathrm{s}}$ then turn on the smoke detection indicator (LED). In this process we get noticed that our smoke detector found the presence of smoke. The output voltage can be generated from the Equation (2)

$$
V_{\text {out }}= \begin{cases}V_{\mathrm{S}+} & V_{1}>V_{2} \\ V_{\mathrm{S}-} & V_{1}<V_{2}\end{cases}
$$

(Where $V_{\mathrm{s}}$ is the supply voltage and the op-amp is powered by $+V_{\mathrm{s}}$ and $-V_{\mathrm{s}}$.)

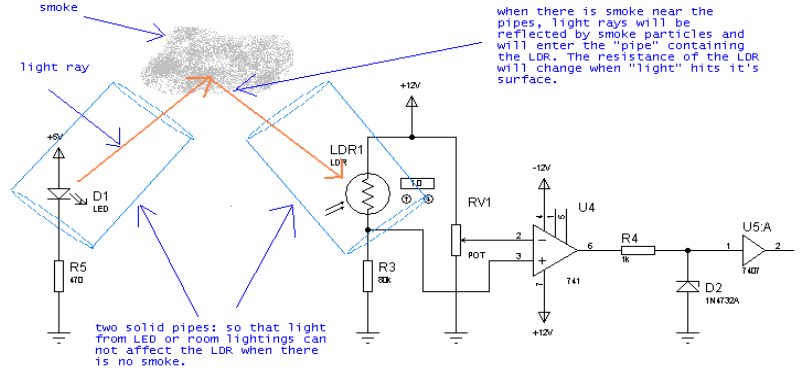

Fig. 4. Schematic view of Smoke Detection Module

Figure 5 shows the operation of zener diode, comparator and potentiometer in proposed Smoke detection module (SDM). In our proposed SDM we used a $5.1 \mathrm{~V}$ zener diode as we need a $5 \mathrm{~V}$ input for the micro controller. This $5.1 \mathrm{~V}$ zener diode will exhibit a voltage drop of $5.1 \mathrm{~V}$ if reverse bias voltage applied across it is more than its zener voltage. From the comparator when we get a + Vs voltage then zener diode makes sure a 5.1 $\mathrm{V}$ output as it exceed the zener voltage. As zener diode has low impedance excessive voltage may obliterate the diode and the whole process. So here we use some resistance for saving the zener diode from excessive voltage. Now we get almost suitable voltage value for the micro controller but not the exact one. For getting the exact one we have to use a Digital Buffer IC.

A Digital Buffer is a single input device that performs any type of logical operation on its input signal as its output exactly matches that of its input signal [7]. It is a "Non-inverting" device and so will give us the Boolean expression. For the suitable value $(5 \mathrm{~V}$ and 0 V) we used a Digital Buffer IC (7407) which is capable for doing these operations. As a simple function this Buffer IC converts the $5.1 \mathrm{~V}$ to the closest value of $5 \mathrm{~V}$ \& $-0.6 \mathrm{~V}$ to the closest value of $0 \mathrm{~V}$ and also represents it into the logical form of operation. The micro controller gets a logical ' 0 ' which represent the 'Absence of Smoke' and gets a logical ' 1 ' which represents the 'presence of Smoke'.

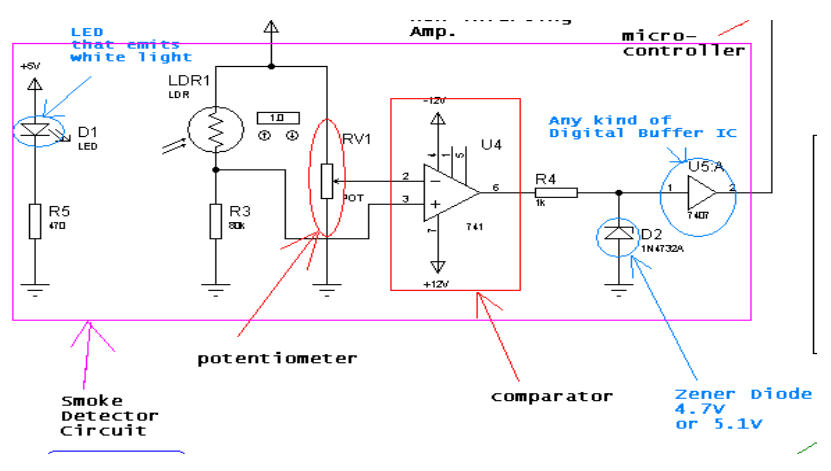

Fig. 5. A closer look into Smoke Detection Module (SDM) 


\subsection{3: Automatic Action Module (AAM)}

In our proposed system Automatic Action Module (AAM) is capable of generating water/gas into the fire to minimize the fire in early stages. We used steeper motor for this purpose. A stepper motor converts electrical pulses into specific rotational movements [8]. The movement created by each pulse is precise and repeatable, which is why stepper motors are so effective for positioning applications [9].

Stepper motors have multiple "toothed" electromagnets arranged around a central gear-shaped piece of iron. The electromagnets are energized by an external control circuit, such as a microcontroller. Stepper motor consists of four coils. At a time when a single coil is excited, the coil creates a magnetic field and the conductor aligns with that coil [10].

Figure 6 shows the mechanism of stepper motor in our proposed system. To excite a coil, we have to put one end of the coil at $+\mathrm{Vs}$ (the source voltage) and the other end at ground $(0$ Volt $)$. In this module, the common wire is kept at $+\mathrm{Vs}$ and the other terminals (A, $\mathrm{B}, \mathrm{C}, \mathrm{D})$ are connected to the ground one at a time. This means when the A terminal is connected to the ground other terminals are open circuited. To implement this idea we used four MOSFETs which acts as a voltage controlled switch. When the gate voltage is $5 \mathrm{~V}$, the switch closes and shorts D and S. Otherwise there is an open circuit between D and S. Micro controller serially provides a series of pulses from the pins PD0, PD1, PD2 and PD3.

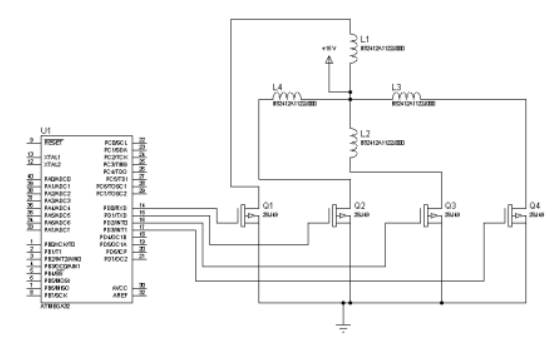

Fig. 6. Operation of Stepper motor

Table 1 show the steeper motor output in AAM. For Type1, the first MOSFET gets a $5 \mathrm{~V}$ at the gate and the other MOSFETs get $0 \mathrm{~V}$. By doing this, the first coil gets excited and the conductor aligns with the first coil. Similarly Type 2, Type 3 and Type 4 and MOSFET 2, MOSFET 3 and MOSFET 4 are on which makes the second, third and fourth coils get excited. Controlling the stepper motor enable the system to provide water/gas in the incident to prevent the fire being spreading.
Table 1: Stepper motor output

\begin{tabular}{|c|c|c|c|c|}
\hline & A & B & C & D \\
\hline Type 1 & 1 & 0 & 0 & 0 \\
\hline Type 2 & 0 & 1 & 0 & 0 \\
\hline Type 3 & 0 & 0 & 1 & 0 \\
\hline Type 4 & 0 & 0 & 0 & 1 \\
\hline
\end{tabular}

\subsection{4: Notification Module (NM)}

In our proposed system the Notification Module (NM) is responsible of generating short message service (SMS) to notify the nearby residents and Fire Fighting team about the incidents. It will also provide the exact location of the incidents by the help of GSM modem. An external GSM modem is connected to the microcontroller through a serial cable or a USB cable [11]. It operates at either $900 \mathrm{MHz}$ or $1800 \mathrm{MHz}$ frequency band and it can support data transfer of up to $9.6 \mathrm{Kbit} / \mathrm{s}$ [12]. In order to operate a GSM modem is also requires a SIM card by which it establishes and manages the connection. The GSM modem comes with a serial interface which the modem can be controlled using AT command interface [13].

GSM modem support a common set of standard AT commands which integrated with the multi controller. After successful connection the program is written in microcontroller to perform the specific task it is up to [14]. The modem produces warning text SMS to the mobile phone of the authority after it receives a command. Getting the SMS will help the authority to act fast and will help to minimize the fire. Depending on the fire the SMS sending process will be differ. When the incident of fire is limited the system will send SMS only those people of that authority but when the situation getting worse then the system will broadcast the SMS to all. For GSM modem a special subscription to an SMS service provider is not required which makes it more cost effective and efficient [15].

In our proposed system it Notification Module (NM) (Figure 7) will consists two types of sensor nodes mobile nodes and Fire station nodes. Data after aggregated in Microprocessor will be relayed to the base stations where the further processing takes place. Base station (BS) relays the information to the operating personnel who takes necessary actions based on the information received. To ensure the location accuracy data from sensors must be combined and dispatched to base station in such a way those nearby sensors should temper their data with each other. To locate the location the device transmits the data to the central server with GPS coordinates using map applications in android mobile through SMS [16] Flow chart of Notification Module (NM) shows how microcontroller integrates with TDM and SDM to generate the operation of AAM and intexgrate GSM/GPRS module to send SMS through BS. 


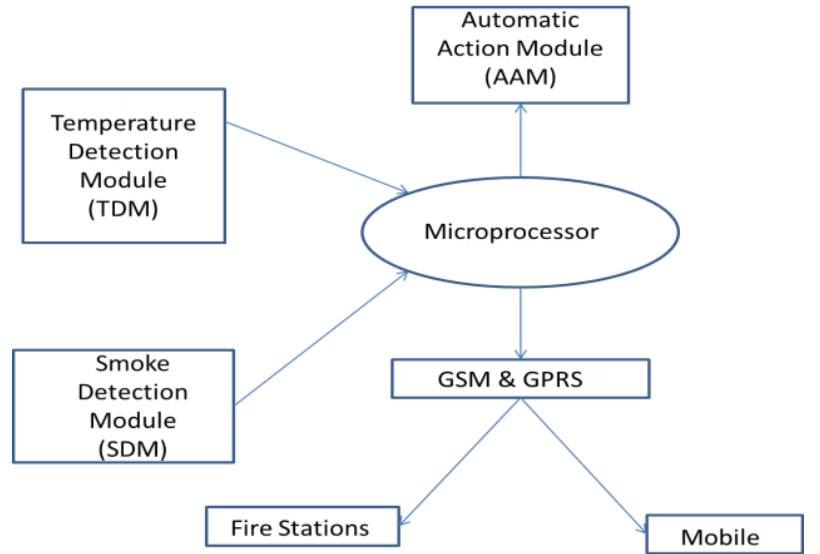

Fig. 7. Flow chart of Notification Module (NM)

\section{Results}

Figure 8, 9 and 10 shows our implemented Temperature Detection Module (TDM), Automatic Action Module (AAM), Smoke detection Module (SDM) and Notification Module (NM) for an Efficient Multi-sensing and GSM Equipped Fire Monitoring System.

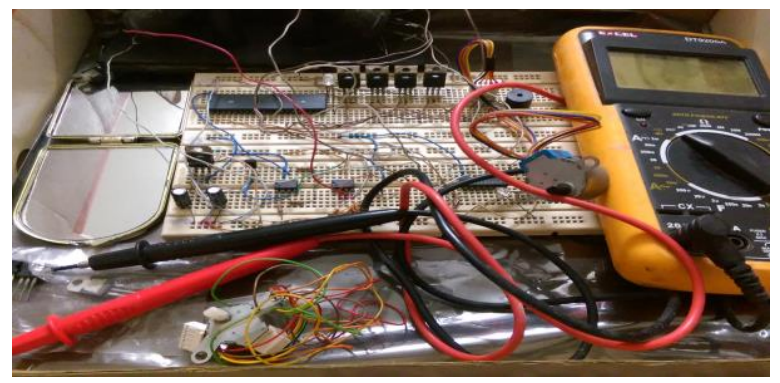

Fig. 8. Temperature Detection Module (TDM) and Automatic Action Module (AAM)

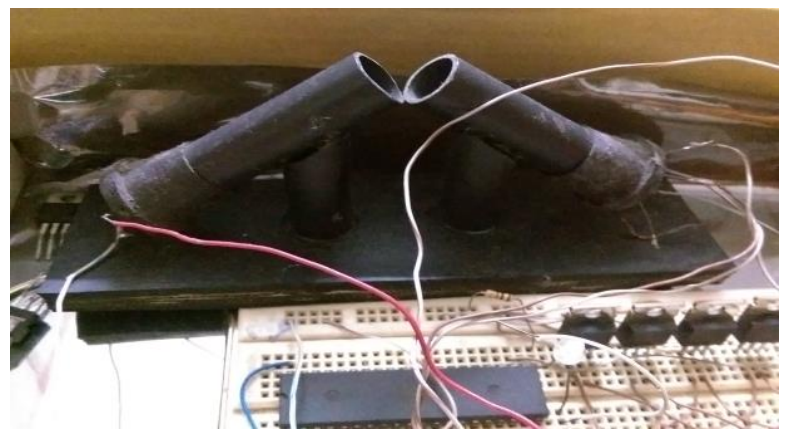

Fig. 9. Smoke detection Module (SDM)

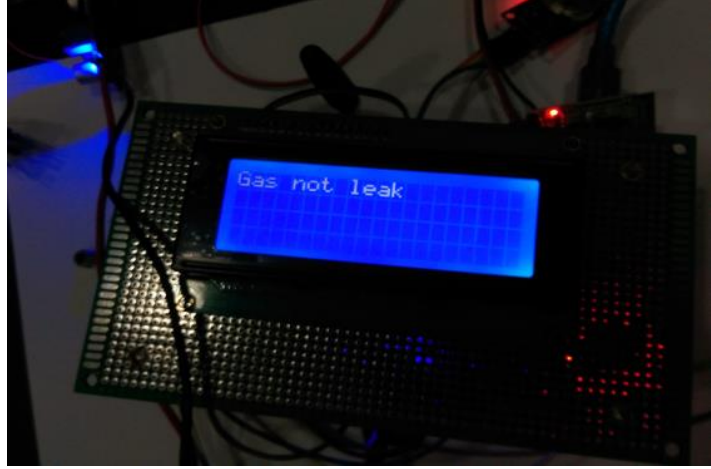

Fig. 10. Notification Module (NM)

\section{Conclusions}

A principal goal of fire monitoring system is to react promptly to a fire and not to misleading particulate signatures produced by nuisance sources. System that only concentrates on smoke detection mechanism could not discriminate between fire and non-fire sources of smoke. Early detection and quick response is the key to minimize damage caused by fires [17]. By using multisensor detectors we can analyze various aspects of a wide selection of signatures produced by flaming fires, non-flaming fires, and nuisance sources [18]

The use of micro controller will bring more intelligence in the system as it now able to distinguish different aspect and can make the best decision out of that. By using GSM modem we not only able to locate the incident spot but also able to warn local authority for quick response [19]. A better understanding on those factors that influence the performance of a fire monitoring system can make a big difference. The outcome from this project can add value to various aspects in manufacturers, designers, authorities, programmer to create further improvement in this sector. As a future work the presence of microcontroller and GSM device will enable the proposed system to generate real time data to monitor the system upfront.

\section{References}

[1] L. Liu, R. Sun, Y. Sun, S. Al-Sarawi, "A smart bushfire monitoring and detection system using GSM technology," International Journal of Computer Aided Engineering and Technology, 2010, 2(2/3), pp. 218-232.

[2] K. V. Teja and S. Angadi, "Fire Detection and Notification System in Trains," International Journal of Innovative Research in Science, Engineering and Technology (IJIRSET), vol. 2(4), April 2013, 11491152.

[3] Y. Yao, J. Yang, C. Huang and W. Zhu, "Fire Monitoring System Based on Multi-Sensor Information Fusion," 2010 2nd International Symposium on Information Engineering and Electronic Commerce, Ternopil, 2010, pp. 1-3.

[4] K Gaikwad, A. Quadri, S. Akshada, Z. Reshma; "Fire Monitoring and Control System," in International 
Research Journal of Engineering and Technology (IRJET), 3(5), 2016.

[5] W. Ping-ping, S. Feng-jie, C. Weixin. "Discussion on temperature monitoring system for power cable joints". Telecommunication for Electric Power System, 27(160), 2006, pp. 59-61.

[6] W. Chao, P. Zhen-cun. "Power Cable Junction Monitoring System for Fault Pre-Warning," Electrical Power Automation Equipment, 21(5), 2001, pp. 25-28.

[7] L. Jun, Q. Chengshan, S. Peng, W. Zhiwei. "Power Cable Connector Monitoring System Based on Wireless Sensor Network Monitoring System," Chinese Journal of Electron Devices, 37, 2014, pp. 766-771.

[8] S. Vitusutrapoj "Advance Microcontroller 8051 Series" Seed Education Co,

[9] Douglas V., "Hall Microprocessors and Interfacing (Programming \& Hardware)", 1991, Glencoe McGrawHill.

[10] J. E. Traister, (2nd edition) "Security/Fire Alarm Systems: Design, Installation, and Maintenance", Columbus, OH: McGraw- Hill, 1995

[11] R. Shams, S. Hossain, S. Priyom, N. Fatema, S. R. Shakil, \& M. K. Rhaman, "An automated firefighting system," 2015 12th International Conference on Fuzzy Systems and Knowledge Discovery, FSKD 2015, 23272331.

[12] J. J. Chen, M. X. Lu, T. H. Wu and Y. S. Hwang, "Sub-1-V Fast-Response Hysteresis-Controlled CMOS Buck Converter Using Adaptive Ramp Techniques," in IEEE Transactions on Very Large Scale Integration (VLSI) Systems, 9(21), pp. 1608-1618, Sept. 2013.

[13] Z. Liu and A. K. Kim, "Review of Recent Developments in Fire Detection Technologies," Journal of Fire Protection Engineering, 2(13) pp. 129-151, May $1,2003$.

[14] N. Bouguila and K. Daoudi, "A Statistical Approach for Binary Vectors Modeling and Clustering," Lecture Notes in Computer Science book series (LNCS, volume 5476), pp. 184-195, 2009.

[15] M. Reddy and K.Rao. "Fire Accident Detection and Prevention monitoring System using Wireless Sensor Network enabled Android Application" Indian Journal of Science and Technology, 9(17), 2016.

[16] H. Wang. "Discriminating Fire Detection via Support Vector Machines", Lecture Notes in Control and Information Sciences, 2006

[17] G. Healey, D. Slater, T. Lin, B. Drda and A. D. Goedeke, "A system for real-time fire detection," Proceedings of IEEE Conference on Computer Vision and Pattern Recognition, New York, NY, 1993, pp. 605606.

[18] A. Goswami, T. Bezboruah, K. C. Sarma, "Design of an embedded system for monitoring and controlling temperature and light," IJEER. 2009: 1(1), pp. 27-36.
[19] S. Dhananjeyan, K. Mohana Sundaram, A. Kalaiyarasi, P. G. Kuppusamy, "Design and Development of Blind Navigation System using GSM and RFID Technology," Indian Journal of Science \& Technology, 9(2), PP. 1-5, 2016. 